Check for updates

Cite this: RSC Adv., 2018, 8, 5882

Received 6th January 2018

Accepted 27th January 2018

DOI: $10.1039 / c 8 r a 00157 j$

rsc.li/rsc-advances

\section{The effect of stoichiometry on the structural, thermal and electronic properties of thermally decomposed nickel oxide $\uparrow$}

\begin{abstract}
P. Dubey, ${ }^{a}$ Netram Kaurav, (D) *a Rupesh S. Devan, ${ }^{\text {b }}$ G. S. Okram (D) ${ }^{c}$ and Y. K. Kuo ${ }^{d}$
A thermal decomposition route with different sintering temperatures was employed to prepare nonstoichiometric nickel oxide $\left(\mathrm{Ni}_{1-\delta} \mathrm{O}\right)$ from $\mathrm{Ni}\left(\mathrm{NO}_{3}\right)_{2} \cdot 6 \mathrm{H}_{2} \mathrm{O}$ as a precursor. The non-stoichiometry of samples was then studied chemically by iodometric titration, wherein the concentration of $\mathrm{Ni}^{3+}$ determined by chemical analysis, which is increasing with increasing excess of oxygen or reducing the sintering temperature from the stoichiometric $\mathrm{NiO}$; it decreases as sintering temperature increases. These results were corroborated by the excess oxygen obtained from the thermo-gravimetric analysis (TGA). X-ray diffraction (XRD) and Fourier transformed infrared (FTIR) techniques indicate the crystalline nature, $\mathrm{Ni}-\mathrm{O}$ bond vibrations and cubic structural phase of $\mathrm{Ni}_{1-\delta} \mathrm{O}$. The change in oxidation state of nickel from $\mathrm{Ni}^{3+}$ to $\mathrm{Ni}^{2+}$ were seen in the X-ray photoelectron spectroscopy (XPS) analysis and found to be completely saturated in $\mathrm{Ni}^{2+}$ as the sintering temperature reaches $700{ }^{\circ} \mathrm{C}$. This analysis accounts for the implication of non-stoichiometric on the magnetization data, which indicate a shift in antiferromagnetic ordering temperature $\left(T_{N}\right)$ due to associated increased magnetic disorder. A sharp transition in the specific heat capacity at $T_{\mathrm{N}}$ and a shift towards lower temperature are also evidenced with respect to the non-stoichiometry of the system.
\end{abstract}

\section{Introduction}

As an antiferromagnetic ${ }^{1}$ and Mott-Hubbard insulator, ${ }^{2}$ stoichiometric nickel oxide (NiO) has been the most exhaustively investigated transition metal oxide. It has attracted considerable interest as a relatively low-cost, low-toxicity, and environmentally-friendly material. Such qualities make it an attractive material that is useful in a range of applications, such as transparent conductive film, ${ }^{3}$ chemical sensors ${ }^{4}$ and resistive random access memory. ${ }^{5}$ In addition, it is also a well-studied material as a positive electrode in batteries ${ }^{6}$ and in quantum dot light emitting devices as a hole transport layer. ${ }^{7}$ The performance of these devices depends on conditions of preparations. For example, on changing these conditions, NiO of different stoichiometry $\left(\mathrm{Ni}_{1-\delta} \mathrm{O}\right)$ can be obtained, in which composition ratios between nickel and oxygen are not exactly $1: 1$. Because of excess oxygen and vacancies on Ni site, nickel

${ }^{a}$ Department of Physics, Government Holkar Science College, A. B. Road, Indore 452001, MP, India. E-mail: netramkaurav@yahoo.co.uk

${ }^{b}$ Discipline of Metallurgy Engineering and Materials Science, Indian Institute of Technology Indore, Khandwa Road, Simrol, Indore 453552, India

${ }^{c} U G C-D A E$ Consortium for Scientific Research, University Campus, Khandwa Road, Indore 452001, MP, India

${ }^{d}$ Department of Physics, National Dong Hwa University, Hualien 97401, Taiwan

$\dagger$ Electronic supplementary information (ESI) available: Extra XRD, TGA, FTIR and XPS results. See DOI: 10.1039/c8ra00157j oxide thus becomes a p-type semiconductor. ${ }^{8}$ Thermodynamically, it is found that this nickel vacancy is the most dominant point defect present in the system. ${ }^{9}$ Various characteristics of the nickel oxide such as electrical, ${ }^{10}$ optical ${ }^{11}$ and thermal ${ }^{12}$ properties depend strongly on its stoichiometry. The changes in physical properties are thought to be attributed to the oxidation state of nickel which changes with oxygen concentration, which eventually produces cation vacant nickel oxide. An excess of oxygen decreases with an increase in sintering temperature of the nickel salt. Densities of nickel oxide and activation energy of electrical conductivity decrease with an increase in the excess of oxygen whereas the lattice parameter do not vary. ${ }^{13}$ Isothermal change in electrical conductivity of nickel oxide shows that the decrease in vacancies may be attributed to diffusion of vacancies to the crystal surface. ${ }^{14}$

Depending on the conditions of preparation, the sintering temperature, in particular, NiO samples of various surface areas, color, and degree of non-stoichiometry can be prepared. Nickel oxide can easily be prepared via several methods, including chemical route, evaporation, ${ }^{7}$ sputtering, ${ }^{15,16} \mathrm{chem}-$ ical deposition, ${ }^{17-19}$ oxidation of nickel, ${ }^{20}$ sol-gel method ${ }^{21}$ and thermal decomposition..$^{22}$ In particular, thermal decomposition method is a broad category for its powder preparation that impacts on other techniques and is an important method in its own, because it is a simple, low-cost and fast endothermic process. Therefore, the present compound $\mathrm{NiO}$ provides a unique example of a non-stoichiometric compound. In the 
present study, we have investigated the effect of oxygen concentration in nickel oxide prepared by the thermal decomposition method. Excess oxygen was estimated by iodometric titration, which was corroborated by thermo-gravimetric analysis (TGA). It is found that the amount of excess oxygen or oxidation state of nickel in nickel oxide is closely related to synthesis temperature. Fourier transforms infrared (FTIR) spectroscopy is used to analyze the bonding of oxygen with metal ions as well as stoichiometry of the prepared samples. Using X-ray Photoelectron Spectroscopy (XPS) as a surface analytical method, binding information, chemical nature and valence states with compositional changes in the samples were investigated. Temperature dependence of magnetic susceptibility $(\chi)$ and heat capacity $\left(C_{\mathrm{p}}\right)$ was then studied to analyze the effect of non-stoichiometry on transition temperature in $\mathrm{Ni}_{1-\delta} \mathrm{O}$ samples.

\section{Experimental}

\subsection{Synthetic of non-stoichiometric nickel oxide}

Non-stoichiometric nickel oxide was obtained by thermal decomposition of nickel nitrate hexahydrate. ${ }^{23,24}$ Typically, about $5 \mathrm{~g} \mathrm{Ni}\left(\mathrm{NO}_{3}\right)_{2} \cdot 6 \mathrm{H}_{2} \mathrm{O}$ was decomposed thermally in open air for 3 hours at $400{ }^{\circ} \mathrm{C}$ to produce nickel oxide sample with a particular content of oxygen $\left(\mathrm{Ni}_{1-\delta} \mathrm{O}\right)$. The product thus obtained was pure, as no catalyst is required in this method. The precursor breaks down in two or more products in the process in which the gaseous parts are escaped from the system freely. The kinetics of this process is such that it reveals the minimum time-temperature condition necessary for the decomposition. This sample was denoted as NiO400. Similarly, seven other samples were prepared at $500{ }^{\circ} \mathrm{C}, 600{ }^{\circ} \mathrm{C}, 700{ }^{\circ} \mathrm{C}, 800{ }^{\circ} \mathrm{C}, 900{ }^{\circ} \mathrm{C}$, $1000{ }^{\circ} \mathrm{C}$ and $1100{ }^{\circ} \mathrm{C}$. They were denoted NiO500, NiO600, NiO700, NiO800, NiO900, NiO1000 and NiO1100, respectively.

The Bruker D8 Advance X-ray diffractometer with $\mathrm{Cu} \mathrm{K \alpha}$ radiation $(0.154 \mathrm{~nm})$ in the angle range $10-90^{\circ}$ was used for the laboratory method of XRD measurements of the samples in powder form. X-rays were detected using a fast counting detector based on Silicon Strip Technology (Bruker Lynx Eye detector). To determine oxygen content, iodometric (redox) titration was used with standardized sodium thiosulfate and potassium iodide solution with a starch solution as an endpoint indicator. Thermo-gravimetric analysis (TGA) was used to further corroborate the non-stoichiometry in these samples. The TGA system with the top of the line METTLER TOLEDO ultra-micro balance with unique built-in calibration weights ensures an accuracy of $0.1 \mu \mathrm{g}$.

In the Fourier transform infrared (FTIR) transmission measurements, a few micrograms of each sample were added to a fixed quantity of pure $\mathrm{KBr}$ and ground thoroughly. Then, a pellet of the uniform mixture was made for each sample. FTIR spectrum of a pure KBr pellet was subtracted from the FTIR data of each sample to obtain the corresponding FTIR spectra. X-ray photoelectron spectroscopy (XPS) measurements were done on a PHI 5600 CI (Physical Electronics) spectrometer using $\mathrm{Al} \mathrm{K \alpha}$ non-monochromatic X-ray excitation at $350 \mathrm{~W}$ power, an analysis area of $0.8 \mathrm{~mm}$ in diameter and pass energy of $200 \mathrm{eV}$ for electron analysis. The experimental error was below $0.1 \mathrm{eV}$ while the spectrometer resolution was better than $1 \mathrm{eV}$. These data were used for identifying the compositions of $\mathrm{Ni}_{1-\delta} \mathrm{O}$ samples. Magnetization measurements were done on VSM-SQUID (Quantum Design) with a maximum of 7 tesla field. Specific heat is obtained with modulated differential scanning calorimeter MDSC-2910 (TA Instruments).

\section{Results and discussion}

\subsection{X-ray diffraction analysis}

Fig. 1 and $\mathrm{S} 1$ in $\mathrm{ESI} \dagger$ shows XRD patterns of $\mathrm{Ni}_{1-\delta} \mathrm{O}$ samples, NiO400, NiO500, NiO700, NiO1100 and NiO600, NiO800, NiO900, NiO1000. XRD peaks match well with the standard XRD of NiO (JCPDS 47-1049) with no other impurity peaks, showing that these samples were of a single phase in nature in each of them. XRD patterns were profile-refined using the Full-Prof software package [http://www-llb.cea.fr/fullweb/]. Fig. 2 gives the representative Rietveld profile fit for $\mathrm{NiO} 400$ and NiO700 samples along with different patterns obtained by using Fm3m (225) space group. In overall, the XRD analysis shows that these samples remain single phase, i.e., NiO face-centre-cubic (fcc) phase. The cell parameters of all samples listed in Table 1. Interestingly, the unit cell volume decreases as the excess oxygen of the sample decreases XRD patterns show diffraction peaks associated only to $\mathrm{NiO}$, indicating that nickel-nitrate hexahydrate has been transformed mostly into NiO. However, some traces of organic compounds may still be present in samples prepared below $700{ }^{\circ} \mathrm{C}$ as discussed in the later section.

\subsection{Excess oxygen determination}

The changing of nickel ions oxidation state can be realized either by changing sintering temperature ${ }^{25}$ or the composition of a sample by diluting foreign atoms. ${ }^{26}$ In general, one expects an excess of oxygen in $\mathrm{Ni}_{1-\delta} \mathrm{O}$ samples. To determine the

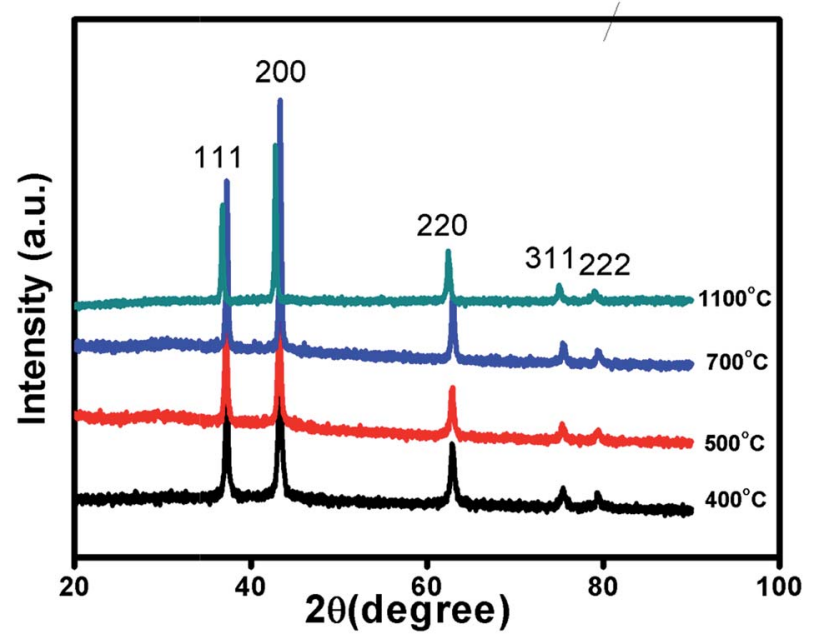

Fig. 1 Characterization of sample. X-ray diffraction pattern of nonstoichiometric $\mathrm{Ni}_{1-\delta} \mathrm{O}$ samples sintered at different temperature as indicated. 


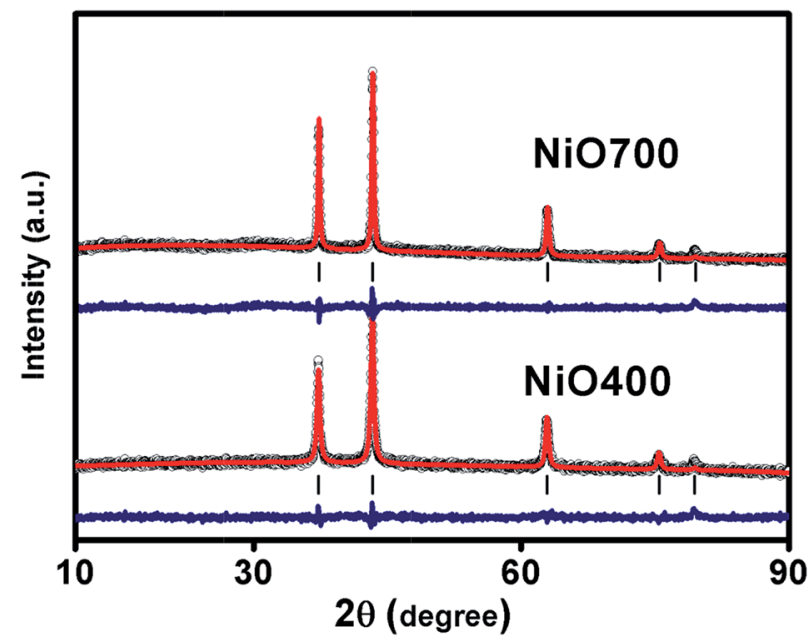

Fig. 2 Shows the graphic results of the representative Rietveld refinement fitting of XRD data for $\mathrm{NiO} 400$ and NiO700. Experimental data (open circles), with Rietveld fitted curve (continuous red), vertical lines indicating peak positions and difference.

content of oxygen in the presently studied $\mathrm{Ni}_{1-\delta} \mathrm{O}$ samples, iodometric titrations were carried out using sodium thiosulfate $\left(2.023 \times 10^{-3} \mathrm{~mol}^{-1}\right)$ as a titrant. Samples ( $c$. $\left.0.025 \mathrm{~g}\right)$ were dissolved in a solution of $\mathrm{KI}$ and $\mathrm{HCl}\left(10.0 \mathrm{ml}\right.$; $c a .0 .1 \mathrm{~mol}^{-1} \mathrm{KI}$, ca. $0.1 \mathrm{~mol}^{-1} \mathrm{HCl}$ ). Resulting solutions were diluted to $25 \mathrm{ml}$ and titrated immediately against thiosulfate solution. The starch indicator was added prior to the end-point being reached. The excess oxygen in $\mathrm{NiO}$ samples prepared at different temperatures is shown in Table 1. It was found that the compositional ratio of $\mathrm{O} / \mathrm{Ni}$ is larger than 1 for samples prepared below $700{ }^{\circ} \mathrm{C}$ and it is nearly 1 for samples prepared above $700{ }^{\circ} \mathrm{C}$.

This finding suggests that the nickel oxide prepared by heating the precursor below $700{ }^{\circ} \mathrm{C}$ has an excess of oxygen and hence the oxidation state of nickel changes with a change in sintering temperature. By iodometric titration, the amount of $\mathrm{Ni}^{3+}$ is also determined. Here, the result is expressed in percent atoms of oxygen in excess, by considering that two $\mathrm{Ni}^{3+}$ ions correspond to three ions of $\mathrm{O}^{2-}$. In order to further estimation of excess oxygen, TGA was performed in the inert atmosphere. In general, TGA is a technique that measures the change in weight of a sample as it is heated, cooled or held at constant temperature, which eventually characterizes materials with regard to their compositions. In the present investigation, the weight of the non-stoichiometric oxygen is obtained directly by obtaining the weight loss. The thermogravimetric (TG) curve and its differential curve (DGA curve-derivatographic analysis) are shown in Fig. 3(a) for NiO400 sample. It is evident from this figure that a very sharp peak is present between 200 and $300{ }^{\circ} \mathrm{C}$ on the DGA curve, which is a measure of the amount of excess oxygen released in the heating process. The corresponding weight loss reflects desorption of oxygen in the nonstoichiometric sample. On the other hand, the peak below $100{ }^{\circ} \mathrm{C}$ is due to the evaporation of the physically bound water in the sample and does not contribute to the estimation of excess oxygen. As shown in Fig. 3(b-d) by TG and DGA curves, it is noted that for NiO500 sample, the reduction of mass with temperatures decreases and becomes negligible for the sample prepared above $700{ }^{\circ} \mathrm{C}$. The weight percentage of excess oxygen is tabulated in Table 2 from above TG curves. It can be seen from the Table 2 that the estimation of excess oxygen in these non-stoichiometric samples by two different methods corroborates with each other. In order to further explore, the essential feature of excess oxygen in these non-stoichiometric samples, we have prepared another NiO400 sample in the presence of oxygen flow during heat treatment. The TG curve and its DGA are shown in Fig. 3(a) and S2 in ESI, $\dagger$ respectively. Both the curves are nearly identical; however, the weight percentage loss is slightly less as compared to pristine NiO400 sample. The difference in loss of weight percentage is calculated and values are reported in Table 1 . This might be due to the differences in the deficiency of nickel in NiO400 samples with and without oxygen annealing during heat treatment. Thus, heating the $\mathrm{Ni}_{1-\delta} \mathrm{O}$ at higher temperatures heals the defects and leads to the corresponding atomic rearrangement. Thus, highly defective nickel oxide at lower temperature approaches stoichiometric nickel oxide as the heating temperature increases, presumably, due to change in oxidation state of nickel as the sintering temperature changes.

\subsection{Spectroscopic analysis}

(a) FTIR study. FTIR spectroscopic study of $\mathrm{Ni}_{1-\delta} \mathrm{O}$ samples provides valuable information about the phase composition and the way in which oxygen is bonded to metal ions. Fig. 4(a-d) show infrared (IR) transmission spectrums of NiO400, NiO500, NiO700 and NiO1100 samples and Fig. S3(a-d) in ESI† shows NiO600, NiO800, NiO900 and NiO1000 having different oxygen

Table 1 Rietveld refinement parameters of nonstoichiometric nickel oxide samples

\begin{tabular}{|c|c|c|c|c|c|c|c|c|}
\hline Samples & $a(\AA)$ & Volume $\left(\AA^{3}\right)$ & $R_{\mathrm{f}}$ factor & Breggs, $R$-factor & $R_{\mathrm{p}}$ & $R_{\mathrm{wp}}$ & $R_{\mathrm{e}}$ & $\chi^{2}$ \\
\hline NiO400 & $4.1764( \pm 0.000934)$ & $72.8460( \pm 0.028)$ & 7.15 & 3.94 & 24 & 16.7 & 14.9 & 1.261 \\
\hline NiO500 & $4.1745( \pm 0.000)$ & $72.7467( \pm 0.000)$ & 8.01 & 4.33 & 29.6 & 19.6 & 16.7 & 1.381 \\
\hline $\mathrm{NiO} 700$ & $4.1732( \pm 0.000)$ & $72.6787( \pm 0.000)$ & 8.14 & 4.51 & 22 & 15.8 & 13.3 & 1.416 \\
\hline NiO800 & $4.1728( \pm 0.000)$ & $72.6579( \pm 0.000)$ & 8.46 & 8.58 & 27 & 18.4 & 15.6 & 1.397 \\
\hline NiO900 & $4.1721( \pm 0.000521)$ & $72.6213( \pm 0.016)$ & 12.0 & 12.0 & 39.5 & 26.9 & 17.0 & 1.157 \\
\hline
\end{tabular}



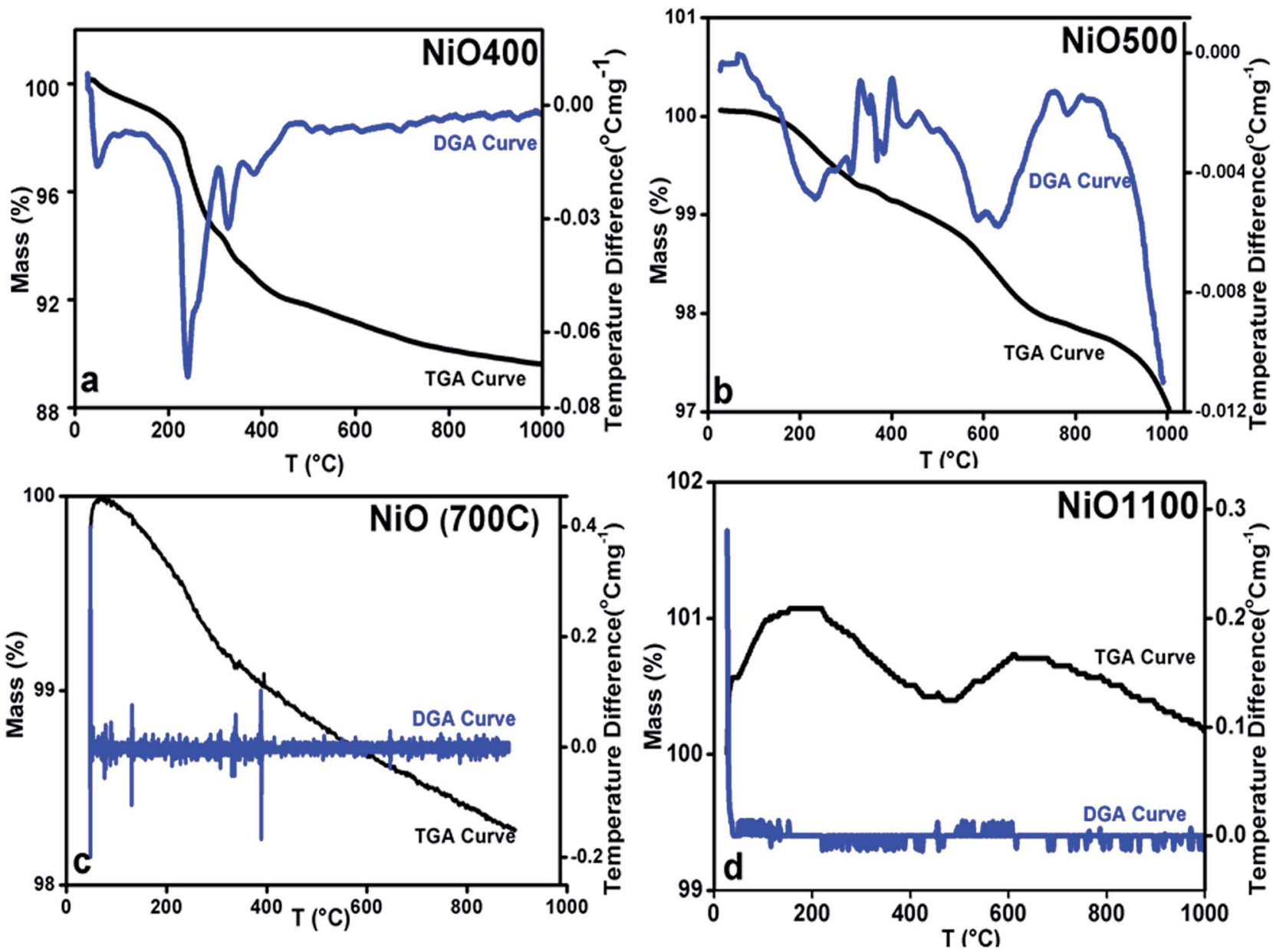

Fig. 3 TGA curves of non-stoichiometric $\mathrm{Ni}_{1-\delta} \mathrm{O}$ samples for (a) $\mathrm{NiO} 400$, (b) $\mathrm{NiO} 500$, (c) $\mathrm{NiO} 700$ and (d) $\mathrm{NiO} 1100$; heating rate of $5{ }^{\circ} \mathrm{C}$ per min was maintained in all.

contents and oxidation state in the range between 400 and $4000 \mathrm{~cm}^{-1}$. The spectrum exhibits a noticeable shoulder peak in the region of $440-460 \mathrm{~cm}^{-1}$. For NiO700 to NiO1100 samples, this peak attains a maximum at nearly $460 \mathrm{~cm}^{-1}$ and a slight shifting of 10 to $20 \mathrm{~cm}^{-1}$ for non-stoichiometric NiO400 to NiO600 samples is noticed. The observation of such peak in the long wavelength region, analogous to previous reports, ${ }^{27}$ could be assigned to the $\mathrm{Ni}-\mathrm{O}$ stretching vibration mode and the shifting is an indication of the non-stoichiometry present in

Table 2 Excess oxygen calculated from iodometric titration and TGA

\begin{tabular}{lll}
\hline Samples & $\begin{array}{l}\text { \% of excess oxygen in } \\
\text { iodometric titration }\end{array}$ & $\begin{array}{l}\text { \% of excess oxygen in } \\
\text { thermogravimetric analysis }\end{array}$ \\
\hline NiO400 & 39.51 & 0.388 \\
NiO500 & 39.46 & 0.388 \\
NiO600 & 39.40 & 0.388 \\
NiO700 & 39.38 & 0.388 \\
NiO800 & 39.40 & 0.388 \\
NiO900 & 39.41 & 0.388 \\
NiO1000 & 39.57 & 0.388 \\
NiO1100 & 39.41 & 0.388
\end{tabular}

these samples. In fact, in this long wavelength transverse optical mode, in which the sublattice of $\mathrm{Ni}^{2+}$ ions moves $180^{\circ}$ opposite to the sublattice of $\mathrm{O}^{2-}$ ions for bulk $\mathrm{NiO}$ has been reported to lie between 390 and $405 \mathrm{~cm}^{-1}$. $^{28}$

There exist other intensive peaks at 1032, 1383 and $1612 \mathrm{~cm}^{-1}$ in $\mathrm{Ni}_{1-\delta} \mathrm{O}$ samples prepared below $700{ }^{\circ} \mathrm{C}$. The existence of these bands clearly indicates the existence of organic molecules such as nitrate ions, water molecules and/or hydroxide ions. It is instructive to mention that the thermal analysis evidently identifies that the $\mathrm{Ni}\left(\mathrm{NO}_{3}\right)_{2} \cdot 6 \mathrm{H}_{2} \mathrm{O}$ was decomposed completely to $\mathrm{NiO}$ at temperatures higher than $600{ }^{\circ} \mathrm{C} .{ }^{24} \mathrm{Hence}$, at the decomposition temperature up to $600^{\circ} \mathrm{C}$, such organic molecules remains in samples leading to the evolution of transmission bands as mentioned above. Further, some of the bands are found to be disappeared as the decomposition temperature increases as TGA data indicts that at higher sintering temperature excess oxygen decreases. ${ }^{24}$ The peaks in IR spectrum at 2924 and $3456 \mathrm{~cm}^{-1}$ could be assigning to the presence of carbon in these samples.

These FTIR data (Fig. 4(a-d)) are thus consistent with those found in TGA (Fig. 3(a-d)) and iodometric analysis data. Therefore, using the vacancy model, we can explain the variation of the hole concentration of nickel oxide. $\mathrm{Ni}_{1-\delta} \mathrm{O}$ is 

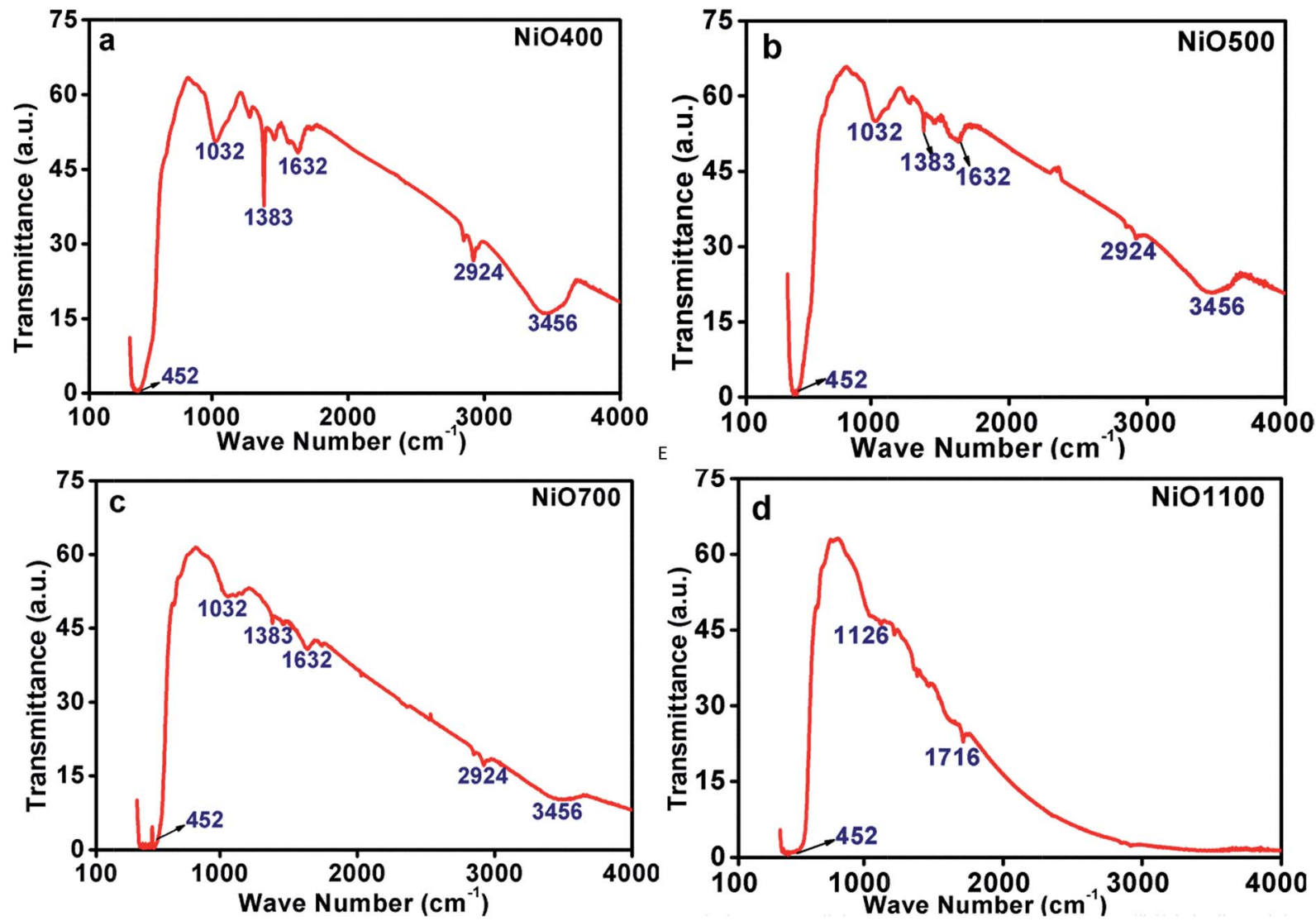

Fig. 4 FTIR of non-stoichiometric $\mathrm{Ni}_{1-\delta} \mathrm{O}$ samples for (a) NiO400, (b) NiO500, (c) NiO700 and (d) NiO1100.

a typically metal-deficient metal oxide. Nickel vacancies are formed at nickel cation sites in NiO due to excess of oxygen ions. The nickel vacancies created at the cation sites can be ionized to create $\mathrm{Ni}^{3+}$ ions via the following reaction ${ }^{29}$

$$
2 \mathrm{Ni}_{\mathrm{Ni}}^{\times}+\frac{1}{2} \mathrm{O}_{2} \rightarrow 2 \mathrm{Ni}_{\mathrm{Ni}}+\mathrm{O}_{\mathrm{O}}^{\times}+V_{\mathrm{Ni}}^{\prime \prime} .
$$

Two $\mathrm{Ni}^{2+}$ ions $\left(\mathrm{Ni}_{\mathrm{Ni}}^{\times}\right)$which react with oxygen produce one ionized nickel vacancy $\left(V_{\mathrm{Ni}}^{\prime \prime}\right)$ and two $\mathrm{Ni}^{3+}$ ions $\left(\mathrm{Ni}_{\mathrm{Ni}}\right)$ in the $\mathrm{NiO}$ crystal. Each created $\mathrm{Ni}^{3+}$ ion serves as donor that donate a hole and also affect the conductive property of nickel oxide. The results of iodometric titration, TGA and FTIR analyses demonstrate that non-stoichiometric nickel oxide is nickel-deficient. Here, according to eqn (1), we argue that an increase in number of nickel vacancies created can induce an increase in the number of $\mathrm{Ni}^{3+}$ ions, which in turn increases the hole concentration of non-stoichiometric $\mathrm{Ni}_{1-\delta} \mathrm{O}$ and it becomes a ptype semiconductor.

In fact, the majority of defects in $\mathrm{Ni}_{1-\delta} \mathrm{O}$ inferred to cationic vacancies compensated by electron holes. This model has been confirmed by a Seebeck coefficient, ${ }^{30}$ electrical conductivity ${ }^{31}$ measurements as well as kinetic measurements of the rate of oxidation of nickel metal to nickel oxide. ${ }^{32}$ This defect processes play a vital role in determining the properties of metal deficit nickel oxides and is strongly influenced by the extent of excess oxygen present in the polycrystalline sample. The atomistic simulations can be done to calculate inter-atomic potentials and a sufficiently large inner region, these methods can produce accurate values of the energies of defect formation, migration, and substitution. ${ }^{33}$ In the present case, the potential describing interionic interactions is represented by ionic pair-wise potentials of the form

$$
\begin{aligned}
\phi(r)= & \sum_{i j} \frac{Z_{\mathrm{m}} e^{2}}{r_{i j}}+\sum_{i j} b \beta_{i j} \exp \left(\frac{r_{i}+r_{j}-r_{i j}}{\rho}\right)+\sum_{i j} c_{i j} r_{i j}{ }^{-6} \\
& +\sum_{i j} d_{i j} r_{i j}^{-8} .
\end{aligned}
$$

here, the first term represents long-range Coulomb, the second term corresponds to Hafemeister and Flygare form of shortrange repulsive energies ${ }^{34}$ and van der Waals multipole are represented by third and fourth terms, respectively. The symbols: $c_{i j}$ and $d_{i j}$ are the van der-Waals coefficients and $\beta_{i j}$ is the Pauling coefficient, respectively. $Z_{\mathrm{m}}$ is the modified ionic charge and parametrically includes the Coulomb screening effect, while $b$ and $r$ are short-range parameters. Thus, the effective interionic potential contains only three free parameters $\left(Z_{\mathrm{m}}, b\right.$, and $\left.r\right)$, which can be determined from the crystal properties. $^{35}$

The short-range potential parameters assigned to each ionion interaction were derived by empirical fitting to observed structural properties. In the context of the columbic term, 
integral ionic charges are presumed, i.e., $2^{+}$for $\mathrm{Ni}$ and $2^{-}$for $\mathrm{O}$, which enables a straight forward definition of hole states as $\mathrm{Ni}^{3+}$ or $\mathrm{O}^{-}$. The deduced potential parameters are listed in Table 3 for all the samples. It is clear from the calculated parameters as the sintering temperature of precursor increases the nature of ordering in systems in which ions occupy sites on a facecentered-cubic (fcc) lattice changes the nearest-neighbor (NN) and next-nearest neighbor (NNN) exchange interactions. This happens in such a manner that the structure of $\mathrm{NiO}$ undergoes a week cubic-to-rhombohedral distortion as a result of the magnetostriction effect in the presence of excess oxygen in the samples. ${ }^{36}$ The NN and NNN exchange interactions and the antiferromagnetic (AFM) structure of $\mathrm{NiO}$ are altered due to the presence of excess oxygen in the samples. Since the radius of $\mathrm{Ni}^{3+}$ ions is smaller than that of $\mathrm{Ni}^{2+}$ and hence the $\mathrm{Ni}^{3+}-\mathrm{O}^{2-}$ bond contributes to the shorter bond distance in those samples whose oxygen content is higher. In due course, interatomic potential parameters and bond length change as the sintering temperature changes, the magnetic ordering transition temperature is expected to change as we will discuss in later sections.

(b) XPS study. XPS studies were performed to investigate the stoichiometry and chemical properties of nickel oxide. Fig. 5(a and b) shows the high-resolution XPS spectra of Ni (2p) core level recorded for the NiO samples decomposed at a temperature of 500 and $700{ }^{\circ} \mathrm{C}$. The high-resolution XPS spectra of the samples decomposed at a temperature of 400 and $1100{ }^{\circ} \mathrm{C}$ are provided in ESI (Fig. S4 in ESI†). All observed XPS spectra illustrated distinct five peaks located at various binding energies. However, double peak features representing the Ni (2p) core levels for $\mathrm{NiO}$ were observed in all samples. For precise determination of the double peak features of $\mathrm{Ni}\left(2 \mathrm{p}_{3 / 2}\right)$ and $\mathrm{Ni}$ $\left(2 \mathrm{p}_{1 / 2}\right)$, the XPS spectra were decomposed using Voigt peak fitting function within the Shirley background. The perfect fit for 8 peaks marked as a, a $\mathrm{a}^{\prime}, \mathrm{b}, \mathrm{b}^{\prime}, \mathrm{c}, \mathrm{c}^{\prime}, \mathrm{d}$, and $\mathrm{d}^{\prime}$ are located at binding energy of $853.7( \pm 0.2), 855.5( \pm 0.2), 860.6( \pm 0.2), 865.9$ $( \pm 0.2), 871.4( \pm 0.2), 873.3( \pm 0.2), 877.9( \pm 0.2)$, and 880.7 $( \pm 0.2) \mathrm{eV}$, respectively. The peaks marked as a, $\mathrm{a}^{\prime}, \mathrm{c}$ and $\mathrm{c}^{\prime}$, represent core levels of $\mathrm{Ni}^{2+}\left(2 \mathrm{p}_{3 / 2}\right), \mathrm{Ni}^{3+}\left(2 \mathrm{p}_{3 / 2}\right), \mathrm{Ni}^{2+}\left(2 \mathrm{p}_{1 / 2}\right)$, and $\mathrm{Ni}^{3+}\left(2 \mathrm{p}_{1 / 2}\right)$, respectively. The decomposed shake-up satellite peaks (marked as b, $\mathrm{b}^{\prime}, \mathrm{d}$ and $\mathrm{d}^{\prime}$ ) were observed at $\sim 7.1( \pm 0.2)$ or $10.2( \pm 0.2)$ eV and $\sim 6.3( \pm 0.3)$ or $\sim 7.1( \pm 0.2)$ eV higher in binding energy than that of $\mathrm{Ni}^{2+}\left(2 \mathrm{p}_{3 / 2}\right), \mathrm{Ni}^{3+}\left(2 \mathrm{p}_{3 / 2}\right), \mathrm{Ni}^{2+}\left(2 \mathrm{p}_{1 / 2}\right)$,

Table 3 Interatomic potential parameter of nickel oxide sintered at different temperatures as discussed in the eqn (2)

\begin{tabular}{llll}
\hline Samples & $b\left(10^{-12} \mathrm{erg}\right)$ & $\mathrm{P}(\AA)$ & $\phi(\mathrm{eV})$ \\
\hline NiO400 & 39.51 & 0.388 & -39.592 \\
NiO500 & 39.46 & 0.388 & -39.602 \\
NiO600 & 39.40 & 0.388 & -39.612 \\
NiO700 & 39.38 & 0.388 & -39.623 \\
NiO800 & 39.40 & 0.388 & -39.612 \\
NiO900 & 39.41 & 0.388 & -39.612 \\
NiO1000 & 39.57 & 0.388 & -39.518 \\
NiO1100 & 39.41 & 0.388 & -39.612
\end{tabular}
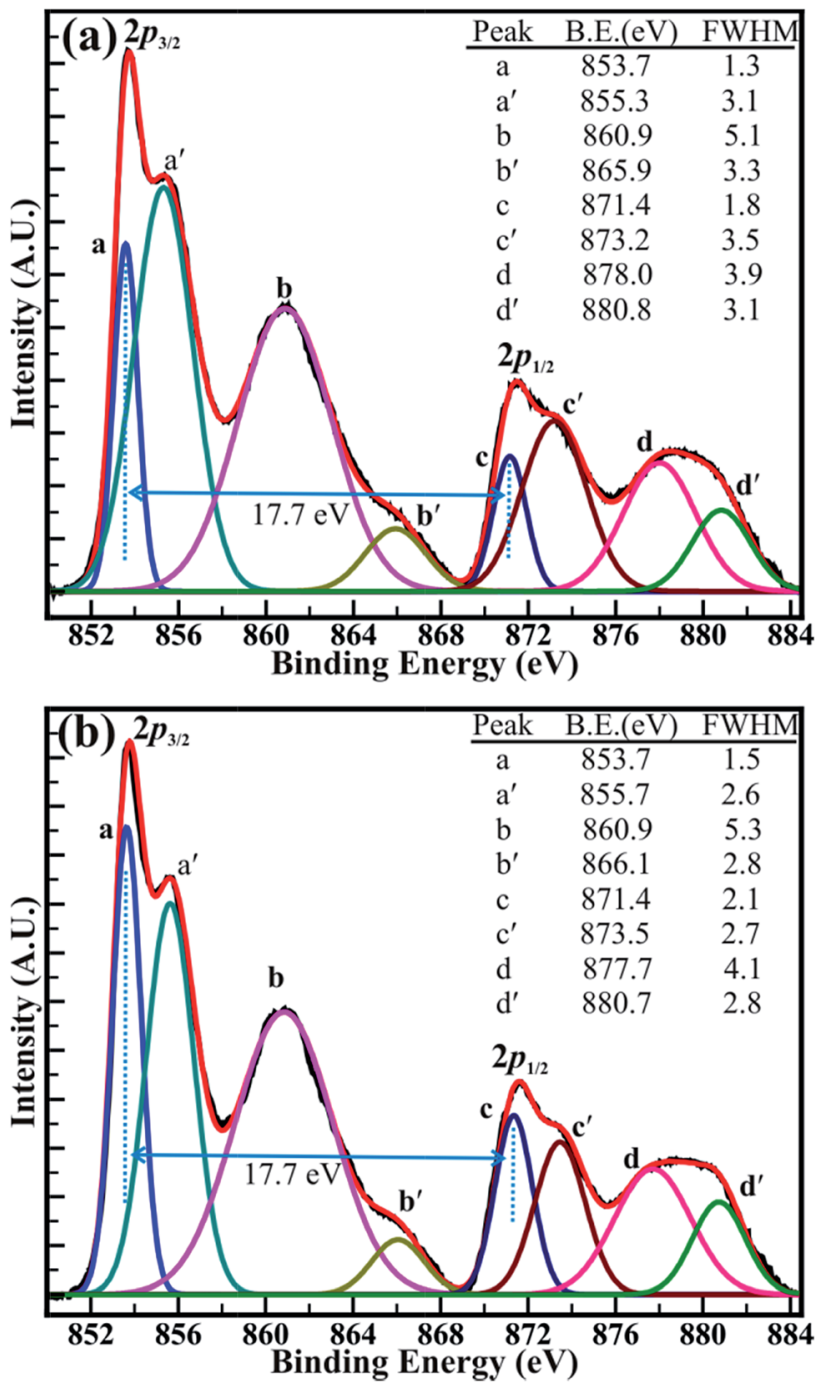

Fig. 5 High-resolution XPS spectra of the $\mathrm{Ni}(2 \mathrm{p})$ core levels of the $\mathrm{Ni}$ oxides decomposed at a temperature of (a) $500^{\circ} \mathrm{C}$ and (b) $700{ }^{\circ} \mathrm{C}$. The XPS spectra were decomposed using Voigt peak function fittings.

and $\mathrm{Ni}^{3+}\left(2 \mathrm{p}_{1 / 2}\right)$ peaks, respectively. The appearance of double peak features of $\mathrm{Ni}(2 \mathrm{p})$ along with their consecutive shake-up satellite peaks are indicative of the magnetic chemical state of $\mathrm{Ni}^{2+}$ and $\mathrm{Ni}^{3+}$ state. ${ }^{37}$ These observations confirm that the peak positions are akin to those reported in previous studies for the presence of $\mathrm{Ni}^{2+}$ and $\mathrm{Ni}^{3+}$ oxidation states in stoichiometric and non-stoichiometric NiO. ${ }^{38}$ Likewise, the representative $\mathrm{O}(1 \mathrm{~s})$ XPS spectra of the sample decomposed at a temperature of $1100{ }^{\circ} \mathrm{C}$ shown in Fig. S5 in ESI $\dagger$ was decomposed using Voigt peak fitting function within the Shirley background. The oxygen spectra show a perfect fit for two peaks located at a binding energy of 529.3 and $531.1 \mathrm{eV}$ with FWHM of 1.2 and $1.7 \mathrm{eV}$, respectively. The lower binding energy peak observed at $529.3 \mathrm{eV}$ corresponds to the $\mathrm{O}(1 \mathrm{~s})$ core level of $\mathrm{O}^{2-}$ anions associated with Ni-O chemical bonding. However higher binding energy peak observed at 531.1 represents the surface contamination or presence of hydroxyl $(-\mathrm{OH})$ groups. ${ }^{38-41}$ 
The close analysis of decomposed XPS spectrum revealed that the intensity of the peaks assigned to core levels of $\mathrm{Ni}^{3+}$ $\left(2 \mathrm{p}_{3 / 2}\right)$ and $\mathrm{Ni}^{3+}\left(2 \mathrm{p}_{1 / 2}\right)$ is larger than that of core levels $\mathrm{Ni}^{2+}\left(2 \mathrm{p}_{3 /}\right.$ 2) and $\mathrm{Ni}^{2+}\left(2 \mathrm{p}_{1 / 2}\right)$ at decomposition temperature of 400 and $500{ }^{\circ} \mathrm{C}$. However, the intensity of the peaks of $\mathrm{Ni}^{2+}\left(2 \mathrm{p}_{3 / 2}\right)$ and $\mathrm{Ni}^{2+}\left(2 \mathrm{p}_{1 / 2}\right)$ core levels has increased than that of peaks of $\mathrm{Ni}^{3+}$ $\left(2 \mathrm{p}_{3 / 2}\right)$ and $\mathrm{Ni}^{3+}\left(2 \mathrm{p}_{1 / 2}\right)$ core levels after the decomposition temperature of $700{ }^{\circ} \mathrm{C}$ and are continued to increase for the temperature of $1100{ }^{\circ} \mathrm{C}$. The intensity ratio obtained for the peaks of the $\mathrm{Ni}^{2+}\left(2 \mathrm{p}_{3 / 2}\right)$ and $\mathrm{Ni}^{3+}\left(2 \mathrm{p}_{3 / 2}\right)$ core levels (i.e. $\mathrm{Ni}^{2+} /$ $\left.\mathrm{Ni}^{3+}\right)$ has increased from $0.85( \pm 0.03)$ to $1.23( \pm 0.03)$ with an increase in the temperature from 400 to $1100{ }^{\circ} \mathrm{C}$. Moreover, the variation in the intensity of the core levels assigned to the $\mathrm{Ni}^{2+}$ and $\mathrm{Ni}^{3+}$ is more apparent at decomposition temperature of 500 and $700{ }^{\circ} \mathrm{C}$ in Fig. $6(\mathrm{a}$ and b). This can be assigned to the transformation of $\mathrm{Ni}^{3+}$ ions into $\mathrm{Ni}^{2+}$ ions with increasing temperature. Furthermore, the binding energy difference $(\Delta E)$ of $17.7( \pm 0.1)$ eV between the $\mathrm{Ni}\left(2 \mathrm{p}_{3 / 2}\right)$ and $\mathrm{Ni}\left(2 \mathrm{p}_{1 / 2}\right)$ peaks is very close to that of $17.8 \mathrm{eV}$ for oxidized $\mathrm{Ni}$ and significantly larger than that of $17.2 \mathrm{eV}$ for metallic $\mathrm{Ni}^{42}$ This confirms again that the ' $\mathrm{Ni}$ ' is materialized in its oxidize forms (i.e. $\mathrm{Ni}^{2+}$, and $\mathrm{Ni}^{3+}$ ) and not in its pure metallic form. Overall, XPS investigation confirms that the non-stoichiometric NiO comprising a higher proportion of $\mathrm{Ni}^{3+}$ ions at $400{ }^{\circ} \mathrm{C}$ has significantly transformed into stoichiometric $\mathrm{NiO}$ at $1100{ }^{\circ} \mathrm{C}$, which is composed of more $\mathrm{Ni}^{2+}$ ions. This analysis from the XPS spectrum shown in Fig. 5(a and b) and S4(a and b) † supports our TGA analysis reported above in this manuscript. Therefore, it can be concluded that the sintering temperature can assist to control the relative concentration of the nickel-oxygen species, distinctly. By controlling the sintering temperature nonstoichiometric nickel oxide can be prepared.

\subsection{Magnetic properties measurements}

The temperature dependence of the magnetic susceptibility $(\chi)$ for selected samples of NiO400 and NiO1100 is plotted in Fig. 6 with an applied magnetic field of 7 tesla. For different

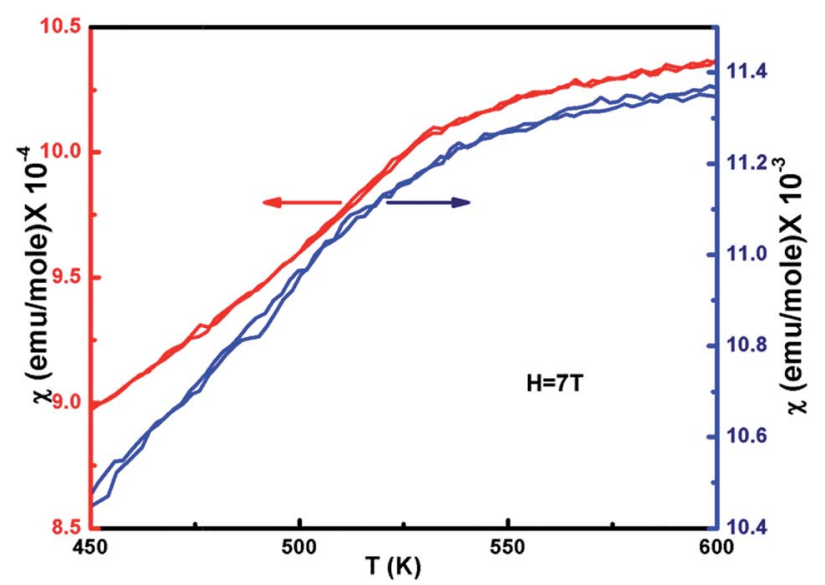

Fig. 6 Magnetization for ZFC and FC curves of non-stoichiometric $\mathrm{Ni}_{1-\delta} \mathrm{O}$ samples for $\mathrm{NiO} 400$ and $\mathrm{NiO} 1100$ in $7 \mathrm{~T}$ applied field as a function of temperature. stoichiometry, the magnetic susceptibility $(\chi)$ values were measured for both the zero-field-cooled (ZFC) and field-cooled (FC) conditions and a reversible behavior with negligible hysteresis of $\chi$ for different stoichiometry was evident. Both samples have paramagnetic (PM) to antiferromagnetic (AFM) transition at the Néel temperature $T_{\mathrm{N}}$ (defined as the slope change in the $\chi v s$. $T$ curve) and the measured values are 480 and $530 \mathrm{~K}$ for NiO400 and NiO1100 samples, respectively. It can be seen from the figure that both the $T_{\mathrm{N}}$ and the absolute value of $\chi$ for NiO400 sample are lower than that of NiO1100 sample. However, PM to AFM transition width appears to be broader for the NiO1100 sample. We argue that the enhancement in $T_{\mathrm{N}}$ with stoichiometry could be attributed to the effect of the partial destruction of $\mathrm{Ni}^{2+}-\mathrm{O}-\mathrm{Ni}^{3+}$ exchange interaction network because of the reduction of the oxygen vacancies and the weakening of $\mathrm{Ni}^{2+}-\mathrm{O}^{2} \mathrm{Ni}^{3+}$ interaction arising from the decrease of the bandwidth of $\mathrm{e}_{g}$ electrons due to the change in $\mathrm{Ni}-\mathrm{O}$ bond length and $\mathrm{Ni}-\mathrm{O}-\mathrm{Ni}$ bond angle. ${ }^{43}$

\subsection{Specific heat measurement}

Specific heat measurements $\left(C_{\mathrm{p}}\right)$ for various samples of nickel oxide for different stoichiometry are presented in Fig. 7. The specific heat anomaly is evident in the vicinity of $T_{\mathrm{N}}$, a clear indication of the AFM ordering in these samples. As the stoichiometry of sample changes, a significant change in the anomaly is observed. Further, a gradual increase in transition temperature from NiO400 to NiO1100 samples is evident as the sintering temperature increases. The obtained values of transition temperature are 510, 519 and $525 \mathrm{~K}$ for NiO500, NiO700 and NiO1100 samples, respectively. It can be seen that due to the presence of excess oxygen in the different ratio in NiO400 and NiO500 samples, change in specific heat anomaly is observed. Further, as the excess oxygen almost disappears in the high temperature sintered samples, the observed transition temperature reaches $525 \mathrm{~K}$ as reported for stoichiometric $\mathrm{NiO}^{44}$ Such an anomalous behavior in $C_{\mathrm{p}}$ at $T_{\mathrm{N}}$ is due to Ni-spin

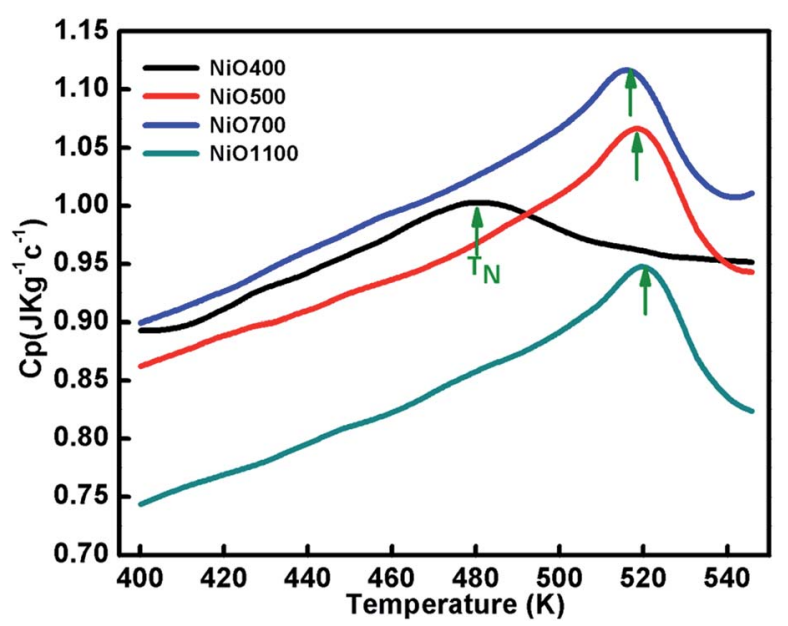

Fig. 7 The temperature variation of specific capacity of nickel oxide samples sintered at different temperatures. Curve shows shifting in $T_{N}$ due different oxygen contents of the samples. 
ordering as suggested by Néel, ${ }^{45}$ wherein the spin-lattice of the particle could reverse coherently and randomly under thermal activation. Consequently, the net moment of uncompensated surface spins would fluctuate accordingly and in turn, significant downshift the $T_{\mathrm{N}}$ in samples due to both the change of magnetic ions as well as their disorder. Presumably, a small amount of excess oxygen could increase magnetic inhomogeneity in samples and eventually shift the Néel temperatures.

\section{Conclusions}

Nickel oxide samples of different stoichiometry were prepared by thermal route method and samples were thoroughly characterized by XRD indexed by full-proof refinement. Their nonstoichiometry was established by iodometric titration and TGA, and the excess oxygen of samples was estimated. The FTIR studies indicated the presence of NiO phase with some amount of hydration and nitrate ions. It also confirms the nonstoichiometry of samples. XPS results revealed that nickel vacancy can be created in samples with varying sintering temperatures. Besides, an excess of $\mathrm{Ni}^{3+}$ ions was noticed in samples sintered at lower temperatures. The temperature variation of $\chi$ for ZFC and FC curves is nearly identical to each sample, while the magnitudes of $\chi$ differ for $\mathrm{Ni}_{1-\delta} \mathrm{O}$ samples with different values of $x$. The observed specific heat anomaly in the vicinity of $T_{\mathrm{N}}$ is associated with the magnetic ordering, indicating a gradual transformation between two magnetic phases and the observed $T_{\mathrm{N}}$ shifted towards lower temperatures as excess oxygen content increases. The shifting of Néel temperature is presumably due to magnetic inhomogeneity arising from the excess oxygen in samples.

\section{Conflicts of interest}

There are no conflicts to declare.

\section{Acknowledgements}

The authors would like to thank Dr A. M. Awasti, Dr Mukul Gupta, and Dr V. Ganesan, Centre Director, UGC-DAE-CSR, Indore, India for providing heat capacity measurements and XRD facilities. Financial support from the Science and Engineering Research Board (SERB), the Department of Science and Technology (DST), New Delhi, the Madhya Pradesh Council of Science and Technology (MPCST), Bhopal (NK), and the Ministry of Science and Technology of Taiwan under Grants No. MOST-103-2112-M-259-008-MY3 (YKK), are gratefully acknowledged.

\section{References}

1 I. Sugiyma, N. Shibata, Z. Wang, S. Kobayashi, T. Yamamoto and Y. Ikuhara, Nat. Nanotechnol., 2013, 8, 266.

2 A. G. Gavriliuk, I. A. Trojan and V. V. Struzhkin, Phys. Rev. Lett., 2012, 109, 086402.

3 H. Sato, T. Minami, S. Takata and T. Yamada, Thin Solid Films, 1993, 27, 236.
4 I. Hotovy, J. Huran, L. Spiess, S. Hascik and V. Rehacek, Sens. Actuators, B, 1999, 57, 147.

5 H. Shima, F. Takano and H. Akinaga, Appl. Phys. Lett., 2007, 91, 012901.

6 Y.-K. Sun, S.-T. Myung, B.-C. Park, J. Prakash, I. Belharouak and K. Amine, Nature, 2009, 8, 320.

7 J. Caruge, J. E. Halpert, V. Bulovic and M. G. Bawendi, Nano Lett., 2006, 6, 2991.

8 A. Nattestad, A. J. Mozer, M. K. R. Fischer, Y.-B. Cheng, A. Mishra, P. Bäuerle and U. Bach, Nat. Mater., 2010, 9, 31.

9 W. Jang, Y.-M. Lu, W.-S. Hwang, T.-L. Hsiung and H. P. Wang, Appl. Phys. Lett., 2009, 94, 062103.

10 P. Kofstad, Nonstoichiometry, Diffusion, and Electrical Conductivity in Binary Metal Oxides, Wiley, New York, 1972.

11 R. S. Conell, D. A. Corrigan and B. R. Powell, Sol. Energy Mater. Sol. Cells, 1992, 25, 301.

12 P. S. Patil and D. S. Kadam, Appl. Surf. Sci., 2002, 199, 211.

13 Y. Iida, S. Kenjo and S. Ozaki, Bull. Chem. Soc. Jpn., 1960, 33, 1372.

14 H.-L. Chen, Y.-M. Lu and W.-S. Hwang, Mater. Trans., 2005, 46, 872 .

15 H. Sato, T. Minami, S. Takato and T. Yamada, Thin Solid Films, 1993, 236, 27.

16 I. Hotovy, D. Bue, S. Hascik and O. Nennewitz, Vacuum, 1998, 50, 41.

17 P. Pramanik and S. Bhattachraya, J. Electrochem. Soc., 1990, 137, 322.

18 A. J. Varkey and A. F. Fort, Thin Solid Films, 1993, 235, 47.

19 M. Chigane and M. Ishikawa, J. Chem. Soc., Faraday Trans., 1998, 94, 3665.

20 C. M. Lampert, T. R. Omstead and P. C. Tu, Sol. Energy Mater., 1986, 14, 161.

21 A. Surca, B. Orel, B. Pilhar and P. Bwkovec, J. Electroanal. Chem., 1996, 408, 83.

22 K. S. Kim and N. Winograd, Surf. Sci., 1974, 43, 625.

23 K. S. Kim and R. E. Davis, J. Electron Spectrosc. Relat. Phenom., 1972, 1, 251.

24 W. Brockner, C. Ehrhardt and M. Gjikaj, Thermochim. Acta, 2007, 64, 456.

25 Y. Iida and S. Ozaki, J. Am. Ceram. Soc., 1959, 42, 219.

26 P. Puspharajah, S. Radhakrishna and A. K. Arof, J. Mater. Sci., 1997, 32, 3001.

27 C. Yu, G. Nazri and C. M. Lampert, Sol. Energy Mater., 1987, $16,1$.

28 I. Nakagawa, Bull. Chem. Soc. Jpn., 1971, 44, 3014.

29 W. J. Moore, Seven Solid States, W. A. Benjamin, Inc., 1967.

30 A. J. Bosman, H. J. Daal, G. F Van and G. F. Knuvers, Phys. Lett., 1965, 19, 372.

31 C. M. Osburn and R. W. Vest, J. Phys. Chem. Solids, 1971, 32, 1331.

32 K. Fueki and J. B. Wegner, J. Electrochem. Soc., 1965, 112, 384.

33 C. R. A. Catlow, Annu. Rev. Mater. Sci., 1986, 16, 517.

34 D. W. Hafemeister and W. H. Flygare, J. Chem. Phys., 1965, 43, 795.

35 N. Kaurav, Phys. Scr., 2013, 88, 015604.

36 N. Mironova-Ulmane, V. Skvortsova and A. Kuzmin, Phys. Solid State, 2005, 47, 1516. 
37 J. S. Shaikh, R. C. Pawar, R. S. Devan, Y. R. Ma, P. P. Salvi, S. S. Kolekar and P. S. Patil, Electrochim. Acta, 2011, 56, 2127. 38 C. D. Wagner, W. M. Riggs, L. E. Davis, J. F. Moulder and G. E. Muilenberg, Handbook of X-ray Photoelectron Spectroscopy, Perkin-Elmer Corporation, Minnesota, 1979.

39 R. S. Devan, V. P. Thakare, V. V. Antad, P. R. Chikate, R. T. Khare, M. A. More, R. S. Dhayal, S. I. Patil, Y.-R. Ma and L. S. Mende, ACS Omega, 2017, 2, 2925.

40 R. S. Devan, Y.-R. Ma, R. A. Patil and S.-M. Lukas, RSC Adv., 2016, 6, 62218.
41 R. S. Devan, Y.-R. Ma, M. A. More, R. T. Khare, V. V. Antad, R. A. Patil, V. P. Thakare, R. S. Dhayalf and L. S. Mendeg, $R S C A d v .$, 2016, 6, 98722.

42 R. S. Devan, C.-L. Lin, S.-Y. Gao, C.-L. Cheng, Y. Liou and Y.-R. Ma, Phys. Chem. Chem. Phys., 2011, 13, 13441.

43 M. Lenglet, F. Hochu, J. Durr and M. H. Tuilier, Solid State Commun., 1997, 104, 793.

44 M. T. Hutchings and E. J. Samuelsen, Phys. Rev. B: Solid State, 1972, 6, 3447.

45 L. Néel, Comptes Rendus, 1961, 252, 4075. 